\title{
Transformation of nomifensine using ionizing radiation and exploration of its anticancer effects in MCF-7 cells
}

\author{
SEONG HEE KANG ${ }^{1 *}$, DONG-HO BAK ${ }^{1 *}$, BYUNG YEOUP CHUNG $^{1}$ and HYOUNG-WOO BAI ${ }^{1,2}$ \\ ${ }^{1}$ Research Division for Radiation Science, Advanced Radiation Technology Institute (ARTI), \\ Korea Atomic Energy Research Institute (KAERI), Jeongeup, Jeollabuk 56212; ${ }^{2}$ Radiation Biotechnology and \\ Applied Radioisotope Science, University of Science and Technology (UST), Daejeon 34113, Republic of Korea
}

Received October 1, 2021; Accepted December 3, 2021

DOI: $10.3892 / \mathrm{etm} .2022 .11235$

\begin{abstract}
Breast cancer is one of the most challenging diseases to treat in humans worldwide. There are several alternatives in treating this life-threatening disease; however, chemoresistance is probably the biggest obstacle to the treatment of breast cancer. It may be essential to develop a therapeutic candidate material with less reversible effects and high treatment efficiency to solve this problem. The present study applied an ionizing radiation approach employing nomifensine (NF) to transform its chemical characteristics and investigated its potential to kill human breast cancer cells (MCF-7). Irradiated (IR-) NF was analyzed using high-performance liquid chromatography. The findings showed that NF inhibited the proliferation of breast cancer cells and increased the rate of apoptosis. In addition, IR-NF induced the accumulation of cytosolic reactive oxygen species and enhanced mitochondrial aggregation. Additionally, mitogen-activated protein kinases (extracellular signal-regulated kinase 1/2, p38 and c-Jun $\mathrm{NH}$ 2-terminal kinase) were involved in damage signaling induced by IR-NF and IR-NF suppressed $\beta$-catenin nuclear translocation. It is suggested that irradiation can be an effective method
\end{abstract}

Correspondence to: DrByung Yeoup Chung or DrHyoung-Woo Bai, Research Division for Radiation Science, Advanced Radiation Technology Institute (ARTI), Korea Atomic Energy Research Institute (KAERI), 29 Geumgu-gil, Jeongeup-si, Jeollabuk 56212, Republic of Korea

E-mail: bychung@kaeri.re.kr

E-mail: hbai@kaeri.re.kr

*Contributed equally

Abbreviations: AKT, protein kinase B; Bcl2, B cell lymphoma 2; ERK, extracellular signal-regulated kinase; GAPDH, glyceraldehyde-3-phosphate dehydrogenase; GSK3 $\beta$, glycogen synthase kinase $3 \beta$; IR-NF, irradiated nomifensine; IR, ionizing radiation; JNK, c-Jun N-terminal kinase; MAPK, Mitogen-activated protein kinase; NF, nomifensine; ROS, Re active oxygen species

Key words: nomifensine, breast cancer, radiolytic transformation, mitogen-activated protein kinase, $\beta$-catenin to maximize the efficacy of existing drugs and that IR-NF has the potential to be a drug candidate for treating patients with breast cancer.

\section{Introduction}

Inhibition and treatment of breast cancer remain a major challenge in the scientific community and the primary goal of breast cancer treatment is to induce cancer cell death and suppress tumor proliferation $(1,2)$. Recently, treatments for inhibiting breast cancer are under several trials for drug resistance and toxicity (3). New therapeutic approaches with improved efficacy and fewer reversible effects are needed to overcome this limitation.

Recently, a report was published on the possible effects of nomifensine (NF) among anticancer substances targeting estrogen signals as a result of large-scale screening in silico (4). Previous research has clearly shown that NF may be an effective new drug targeting breast cancer (5). However, it is necessary to improve its efficacy to lower the reversible effect. NF is a norepinephrine-dopamine reuptake inhibitor that is marketed as an antidepressant (6-8). However, in 1986, the product license for NF $\left(\right.$ Merital $\left.^{\circledR}\right)$ was voluntarily abandoned by the manufacturer for safety reasons (9). In the 1970s, NF was shown to be effective as an antidepressant in doses of 50-225 mg daily (6). However, in the 1980s, psychological dependence on drugs was reported when 400-600 mg was administered daily in patients with a history of stimulant addiction (10). Therefore, to continue using this drug, there is a need for a strategy to reduce the dosage and increase its efficacy.

Several pieces of research have been conducted for increasing the efficacy of biomaterials using radiation transformation technology $(11,12)$. In a previous study, the authors irradiated rotenone with gamma rays and confirmed that its by-product, rotenoisin $\mathrm{B}$, a radiolytic derivative, was produced, which inhibited proliferation in hepatocarcinoma (13). In addition, it was confirmed that radiation-irradiated Kenalog ${ }^{\circledR}$ also increased the apoptotic effect of melanoma compared with Kanalog ${ }^{\circledR}$, the original material (14). To date, there are no reports on the effects of gamma irradiation on the amelioration of the pharmacological effects of NF. The present study used radiation to modify NF and develop a new candidate drug. 
It was hypothesized that NF modified by ionizing radiation (IR)-NF might increase the therapeutic efficacy in inhibiting breast cancer proliferation by inducing cell death.

\section{Materials and methods}

Materials and reagents. All chemicals and reagents were used without further purification. NF and Cell Counting Kit (CCK)-8 were procured from MilliporeSigma. Anti-protein kinase B (AKT; cat. no. 4691), anti-phospho (p)-AKT (cat. no. 4060), anti-glycogen synthase kinase $3 \beta$ (GSK3 $\beta$; cat. no. 9315), anti-p-GSK3 $\beta$ (cat. no. 9322), anti- $\beta$-catenin (cat. no. 8480), anti-BAD (cat. no. 9292), anti-anti-apoptotic B cell lymphoma 2 (Bcl2; cat. no. 2870), anti-caspase 3 (cat. no. 9662), anti-cleaved caspase 3 (cat. no. 9664), anti-cyclin D1 (cat. no. 2978), anti-extracellular signal-regulated kinase (ERK; cat.no. 4695), anti-p-ERK (cat.no. 4377), anti-p38 (cat. no. 9212), anti-p-p38 (cat. no. 9215), anti-c-Jun N-terminal kinase (JNK; cat. no. 9252), anti-p-JNK (cat. no. 9251), anti- $\alpha$-tubulin (cat. no. 2144) and anti-glyceraldehyde-3-phosphate dehydrogenase (GAPDH; cat. no. 2118) were procured from Cell Signalling Technology, Inc. Dulbecco's modified Eagle's medium (DMEM), RPMI 1640, penicillin/streptomycin and foetal bovine serum (FBS) were procured from Lonza Group Ltd. Annexin V and cell cycle kits were procured from MilliporeSigma.

Gamma irradiation and high-performance liquid chromatography (HPLC). A sample solution of NF $(0.5 \mathrm{~g})$ in methanol $(200 \mathrm{ml})$ in chapped vials was irradiated at $0-75 \mathrm{kGy}$ (absorbed dose). Irradiation was carried out at ambient temperature using a cobalt-60 irradiator (point source AECL, IR-79, MDS Nordion International Co. Ltd.) at the Advanced Radiation Technology Institute (Jeongup, Korea). The source strength was $\sim 320 \mathrm{kCi}$ with a dose rate of $10 \mathrm{kGy} / \mathrm{h}$. Using gamma rays, NF in methanol solution was directly irradiated and converted products were monitored using an Agilent Technologies 1100 series HPLC system (Agilent Technologies, Inc.). HPLC analysis was carried out on a YMC-Pack ODS-A column (4.6x150 mm; YMC Korea Co., Ltd.) and was developed at $40^{\circ} \mathrm{C}$ with $1 \%$ formic acid/methyl cyanide (1:1, flow rate: $1.0 \mathrm{ml} / \mathrm{min}$, detection: $280 \mathrm{~nm}$ ). The irradiated methanolic solution was immediately evaporated to remove the solvent and lyophilized.

Cell culture. Human breast cancer MCF-7 cells, human lung cancer A549 cells and human skin cancer SK-MEL-5 cells were procured from American Type Culture Collection. The cells were cultured under sterile conditions at $37^{\circ} \mathrm{C}$ in a humid environment containing $\mathrm{CO}_{2}(5 \%)$ and the culture medium comprised DMEM or RPMI 1640 medium supplemented with FBS (10\%), glutamine (4 mM), penicillin (100 U/ml) and streptomycin $(100 \mu \mathrm{g} / \mathrm{ml})$. The cultures were regularly checked and trypsinised when the cell confluence reached $85 \%$.

Cell viability assay and crystal violet assay. Cell viability was measured using CCK-8 Kit. Cells were seeded in 96-well plates $\left(1 \times 10^{4}\right.$ cells/well) and incubated for $24 \mathrm{~h}$ at $37^{\circ} \mathrm{C}$. The cells were treated with NF or IR-NF $(0,62.5,125 \mu \mathrm{g} / \mathrm{ml})$, and incubated for $24 \mathrm{~h}$ at $37^{\circ}$. A solution of CCK-8 was added to each well and the plates were incubated for $1 \mathrm{~h}$ at $37^{\circ} \mathrm{C}$ to allow the reaction to take place before removal of the culture medium. Cell viability was determined using a spectrophotometer and the absorbance was measured at $450 \mathrm{~nm}$ (Tecan Group, Ltd.). Cell viability for each group was expressed as a percentage of that of the control group. To confirm that the growth of MCF-7 cells was inhibited through induction by IR-NF, a crystal violet assay was performed. Briefly, cells were seeded on sterile coverslips in 12 -well plates $\left(1 \times 10^{4}\right.$ cells/well $)$ and incubated for $48 \mathrm{~h}$. The cells were incubated with NF or IR-NF for $24 \mathrm{~h}$. Thereafter, the cells were stained with $0.1 \%$ crystal violet solution for $20 \mathrm{~min}$ at $37^{\circ} \mathrm{C}$. After discarding the solution, the coverslips were dried, and cells were observed using an inverted phase-contrast microscope (Olympus IX71; Olympus Corporation). The images were captured in 10 randomly selected fields (magnification 10X).

Annexin $V$ assay. The cells $\left(1 \times 10^{6}\right.$ cells/well) grown in a 65-mm culture dish were incubated with NF $(125 \mu \mathrm{g} / \mathrm{ml})$ or IR-NF $(31.2,62.5$ or $125.0 \mu \mathrm{g} / \mathrm{ml})$ for $24 \mathrm{~h}$ at $37^{\circ} \mathrm{C}$. Thereafter, to conduct the quantitative analysis of apoptotic and necrotic dead cells, Muse Annexin V and Dead Cell Assay kits (MilliporeSigma) were used. The cells were harvested and washed with Dulbecco's PBS (DPBS). They were stained with Annexin $\mathrm{V}$ and the Dead Cell reagent for $20 \mathrm{~min}$ at room temperature, and flow cytometric assessment was performed using the Muse Cell Analyser (MilliporeSigma). The number of apoptotic cells was expressed as the percentage of the live, early/late apoptotic and dead cells, which was determined using the Muse version 1.4 analysis software (MilliporeSigma).

Oxidative stress assay. MCF-7 cells ( $2 \times 10^{6}$ cells/well) grown in 12-well plates were incubated with $125 \mu \mathrm{g} / \mathrm{ml}$ of $\mathrm{NF}$ or IR-NF for $24 \mathrm{~h}$ at $37^{\circ} \mathrm{C}$. The oxidative stress assay was conducted by quantitative measurement of cellular populations undergoing oxidative stress using the Muse Cell Analyser and Muse Oxidative Stress kit (MilliporeSigma). According to the manufacturer's protocol, the cells were detached, resuspended to obtain $1 \times 10^{6}$ cells $/ \mathrm{ml}$ and incubated at $37^{\circ} \mathrm{C}$ for $30 \mathrm{~min}$ with the Muse Oxidative Stress working solution. The number of oxidised cells were counted using the Muse Cell Analyser based on the intensity of the red fluorescence. The results were obtained from four independent experiments.

Cell cycle assay. The cells ( $1 \times 10^{6}$ cells/well) grown in a $65-\mathrm{mm}$ culture dish were incubated with $125 \mu \mathrm{g} / \mathrm{ml}$ of NF or IR-NF for $24 \mathrm{~h}$ at $37^{\circ} \mathrm{C}$ prior to IR exposure. Thereafter, to conduct the quantitative analysis of the cell cycle, Muse Cell Cycle Assay kits (MilliporeSigma) were used. The cells were harvested, washed with DPBS and fixed with ethanol $(70 \% \mathrm{v} / \mathrm{v})$. They were stained with Annexin V and the Dead Cell reagent for $20 \mathrm{~min}$ and flow cytometric assessment was performed using the Muse Cell Analyser. The number of apoptotic cells was expressed as the percentage of the live, early/late apoptotic and dead cells, as determined by the Muse version 1.4 analysis software.

Confocal microscopic analysis. The cells ( $1 \times 10^{5}$ cells/well) were prepared on sterilised glass coverslips (BD Biosciences) in triplicate. Cells were incubated with $125 \mu \mathrm{g} / \mathrm{ml}$ of NF or IR-NF 
for $24 \mathrm{~h}$ at $37^{\circ} \mathrm{C}$ prior to IR exposure. i) Immunofluorescence staining: Following irradiation, the cells were fixed in $4 \%$ paraformaldehyde in PBS for $10 \mathrm{~min}$, permeabilized with $0.25 \%$ Triton X-100 in PBS for $10 \mathrm{~min}$ and incubated with the $\beta$-catenin primary antibody for $2 \mathrm{~h}$ at room temperature. The cells were washed to remove the excess primary antibody and incubated with the appropriate fluorescently labelled secondary antibody for $1 \mathrm{~h}$ at room temperature. The nuclei were stained with 4',6-diamidino-2-phenylindole (DAPI) and incubated for $5 \mathrm{~min}$. ii) Annexin V/propidium iodide (PI) staining: Annexin V/PI (5 $\mu \mathrm{l})$ were added to the culture dish and incubated at room temperature in the dark for $10 \mathrm{~min}$. iii) To measure mitochondrial morphological changes, MitoTracker Red CMXRos selective probe (Molecular Probes; Thermo Fisher Scientific, Inc.) were used. The cells were incubated in a medium without FBS and containing $100 \mathrm{nM}$ MitoTracker for $30 \mathrm{~min}$ at $37^{\circ} \mathrm{C}$. The medium was then replaced with a complete medium without MitoTracker, washed twice and the nuclei were stained with DAPI. Fluorescence intensity was analysed for each group. After mounting, fluorescence images were captured using a confocal microscope (LSM 700; Zeiss AG). To quantify the immune-probed cells, the fluorescence intensity was measured in 10 randomly selected images (magnification, $\mathrm{x} 40$ ).

Statistical analysis. Each experiment was performed at least three times and the results were expressed as the mean \pm standard deviation. For multiple comparisons, one-way analysis of variance (ANOVA) was used followed by Tukey's multiple comparisons test. $\mathrm{P}<0.05$ was considered to indicate a statistically significant difference.

\section{Results}

$I R$ altered chemical properties of NF. To detect the chemical structure of NF as changed by IR, NF and IR-NF were dissolved in methanol and monitored by HPLC. Fig. 1A showed that the original peak of NF was lowered and new radiolytic peaks were generated from 12.6 to $15 \mathrm{~min}$. The results indicated that NF transformed into a completely different substance after irradiation. To investigate the physiological activity of IR-NF, newly transformed by IR, three tumor cells were treated with NF and IR-NF for $24 \mathrm{~h}$, following which cell viability was measured. Among the three cells treated with IR-NF, in MCF-7 cells, the cell viability was significantly decreased in the IR-NF-treated group compared with the group treated with the same concentration of NF (Fig. 1B) The reduction reached $92.4 \pm 3.5 \%$ in the $125 \mu \mathrm{g} / \mathrm{ml} \mathrm{NF-treated} \mathrm{group,} \mathrm{whereas} 62.5 \mu \mathrm{g} / \mathrm{ml}$ IR-NF treatment resulted in a reduction in the viability to $39.2 \pm 5.1 \%$ in $\mathrm{MCF}-7$ cells compared with the control. Crystal violet staining results showed that IR-NF inhibited the growth of MCF-7 cells in a concentration-dependent manner. There were no significant differences in A549 and SK-MEL cells (Fig. 1C and D). As shown in Fig. 1E, in the negative control group (Mock) and the NF-treated group, mitosis was actively performed and several colonies were formed. On the other hand, in the cells treated with IR-NF, the relative growth percentage significantly decreased as the treatment concentration increased.
IR-NF induced apoptosis in the MCF-7 cell line. To verify whether the cell growth inhibition affected by NF and IR-NF was induced through apoptosis, Annexin/PI assay and the expression of apoptosis-related proteins were observed. Similar tendencies were observed in the results of Annexin/PI staining (Fig. 2A and B). The total apoptosis rate of the IR-NF-treated group significantly increased from 34.2 to $95 \%$ in a concentration-dependent manner, whereas that of the NF-treated group $(125 \mu \mathrm{g} / \mathrm{ml})$ increased slightly (Fig. 2A). In addition, Fig. 2B shows that Annexin V-positive cells appeared prominently in the IR-NF-treated group. These results suggested that IR-NF has a higher anti-proliferative effect in MCF-7 cells than NF. The present study investigated changes in the $\mathrm{BAD} / \mathrm{Bcl} 2$ ratio following NF or IR-NF treatment. BAD is a representative marker protein for pro-apoptotic proteins, whereas $\mathrm{Bcl} 2$ is a marker protein for anti-apoptotic proteins. It was observed that the ratio of $\mathrm{BAD} / \mathrm{Bcl} 2$ significantly increased in the IR-NF-treated group compared with the control and NF-treated groups (Fig. 2C and D). In addition, as shown in Figs. 2C and D, the expression level of cleaved caspase 3 protein which is a marker protein of programmed cell death, significantly increased compared with that in the NF-treated group. Eventually, it was confirmed that the imbalance of mitochondrial proteins induced by IR-NF treatment induces apoptosis.

IR-NF induced reactive oxygen species (ROS) accumulation and morphological changes of the mitochondria. Further observations were made to confirm whether the cytotoxicity induced by IR-NF was related to excessive ROS production in the cytosol. Mitochondria-derived ROS can mediate redox signalling or, in excess, cause cell injury and even cell death (15). Notably, the number of ROS-positive cells increased after $125 \mu \mathrm{g} / \mathrm{ml}$ of IR-NF treatment, whereas there was no significant change after treatment with the same concentration of NF (Fig. 3A). In addition, aberrant mitochondrial morphology is tightly coupled to excessive ROS accumulation, which may lead to functional damage to the mitochondria. Therefore, mitochondria located around the cell nucleus were also observed after NF or IR-NF treatment. Following the addition of IR-NF, a dense precipitate was observed in the mitochondria and the mitochondria were swollen. These changes were more pronounced in the IR-NF group compared with the NF group (Fig. 3B). These results indicated that IR-NF induces structural and functional damage to the mitochondria, resulting in apoptotic cell death.

The mitogen-activated protein kinase (MAPK) (extracellular signal-regulated kinase 1/2,p38 and c-Jun NH 2-terminal kinase) pathway is involved in cell death-induced by irradiated nomifensine. The stress-responsive MAPK serves an important role in mammalian cells. In particular, MAPK is considered an important prognostic factor for breast cancer (16). Thus, MAPK family proteins (ERK1/2,p38 and JNK) were explored in IR-NF-treated MCF-7 cells using western blotting analysis. As shown in Figs. 4A and B, after the addition of either substance, phosphorylation of JNK and p38 was upregulated, whereas that of ERK was downregulated. These phenomena were more noticeable in the IR-NF-treated group than in the NF-treated group. These results suggest that IR-NF influences 


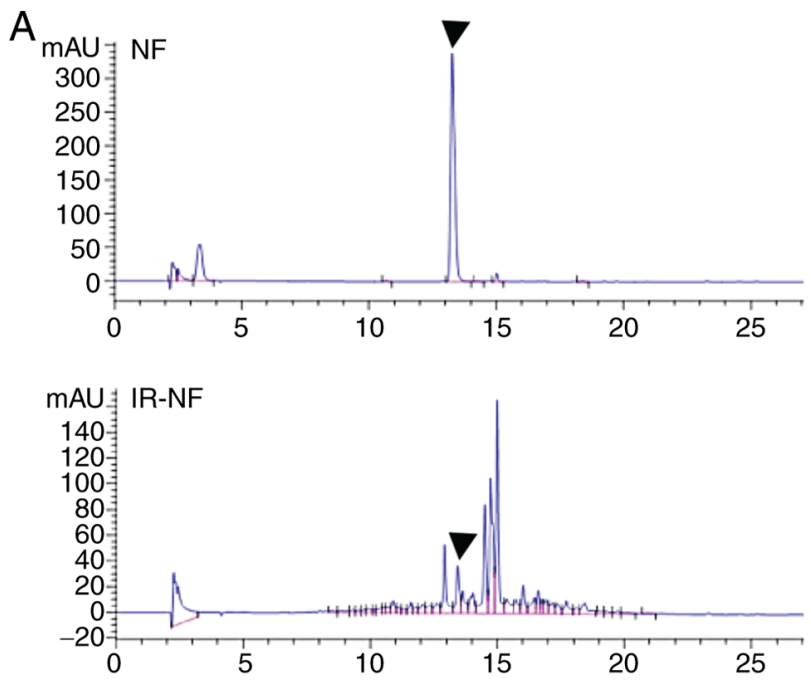

C

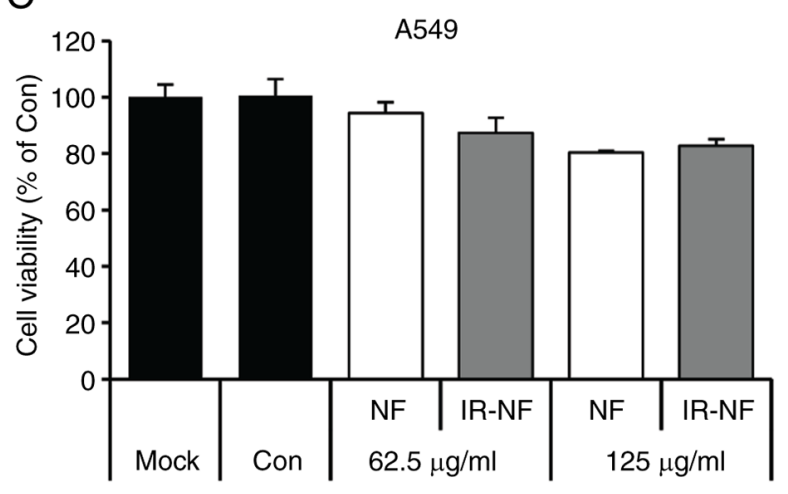

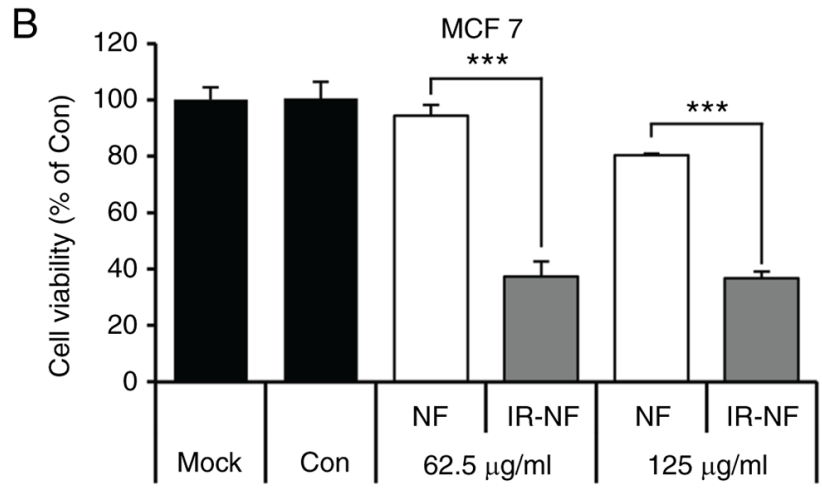

D

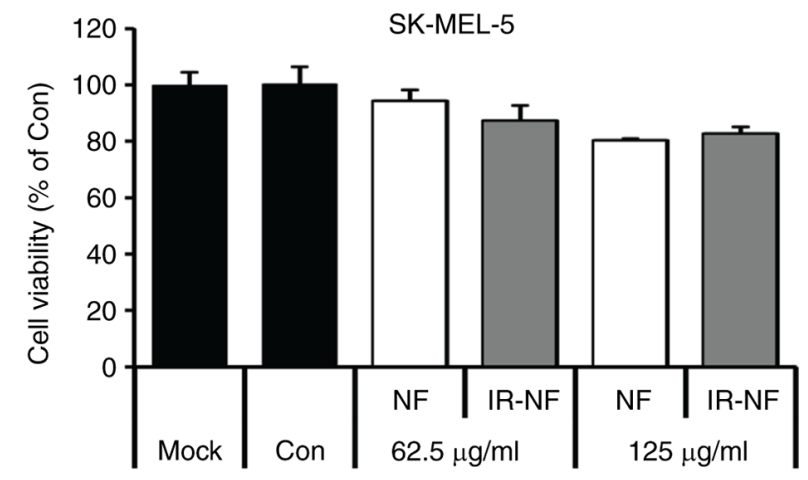

E

MCF7

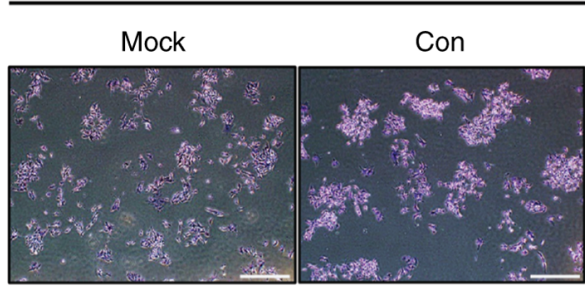
$\mathrm{NF}(125 \mu \mathrm{g} / \mathrm{ml})$ IR-NF $(62.5 \mu \mathrm{g} / \mathrm{ml})$ IR-NF (125 $\mu \mathrm{g} / \mathrm{ml})$

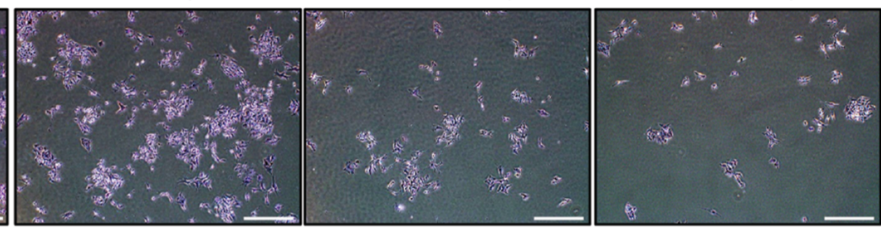

Figure 1. IR altered physiological properties of NF and (IR-NF) inhibits the proliferation of breast cancer (MCF-7) cells. (A) Chromatograms of (top) NF and (bottom) IR-NF. The MCF-7 cells were treated with increasing concentrations of NF or IR-NF in (B) MCF-7, (C) A549 and (D) SK-MEL-5 for $24 \mathrm{~h}$. The effects of IR-NF on the viability of MCF-7 cells at the indicated concentrations were determined using the Cell Counting Kit-8 assay and compared with NF. Data are presented as the means \pm standard deviation of three independent experiments $(\mathrm{P} \leq 0.05)$. (E) Representative images of the morphological changes in MCF-7 cells were observed at $24 \mathrm{~h}$ after NF and IR-NF treatments, respectively. Scale bar=200 $\mu \mathrm{m} .{ }^{* * *} \mathrm{P} \leq 0.001 \mathrm{vs}$. NF. IR, ionizing radiation; NF, nomifensine; IR-NF, irradiated nomifensine; Mock, untreated, Con, vehicle control.

cell death through the regulation of the MAPK (ERK1/2, p38 and JNK) signalling pathways.

IR-NF induced cell cycle arrest and suppressed $\beta$-catenin translocation to the nucleus in MCF-7 Cells. The Wnt/ $\beta$-catenin signalling pathway serves a key role in diverse physiological processes, such as proliferation, differentiation, migration, invasion, apoptosis and tissue homeostasis (17). $\beta$-catenin, a major transcription factor in the $\mathrm{Wnt} / \beta$-catenin signalling pathway, contributes to pivotal molecular mechanisms in cancer development and progression $(18,19)$. In the present study, MCF-7 cells treated with IR-NF showed significant arrest at the $\mathrm{G}_{0} / \mathrm{G}_{1}$ stage (Fig. 5A). Moreover, IR-NF not only downregulated the expression of cyclin D1 (Fig. 5B and C) but also inhibited the nuclear translocation of $\beta$-catenin (Fig. 5D). In addition, IR-NF treatment suppressed its interaction with the AKT/GSK3 $\beta$ cascade (Figs. 5D-F), followed by a decreased $\beta$-catenin expression. Therefore, IR-NF was found to inhibit tumour development and progression by blocking or interfering with the interaction between the Wnt/ $\beta$-catenin and phosphatidylinositol 3-kinase-AKT pathways.

\section{Discussion}

NF was used as an antidepressant; however, its use was discontinued due to a history of drug dependence when the drug was used in very high doses. However, a recent large-scale screening in silico has suggested its pharmacological potential 

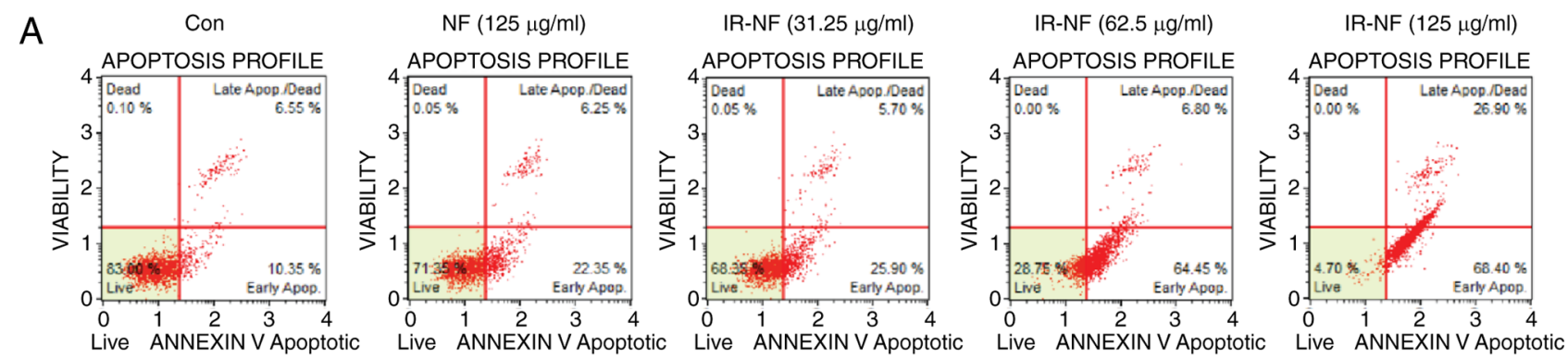

B
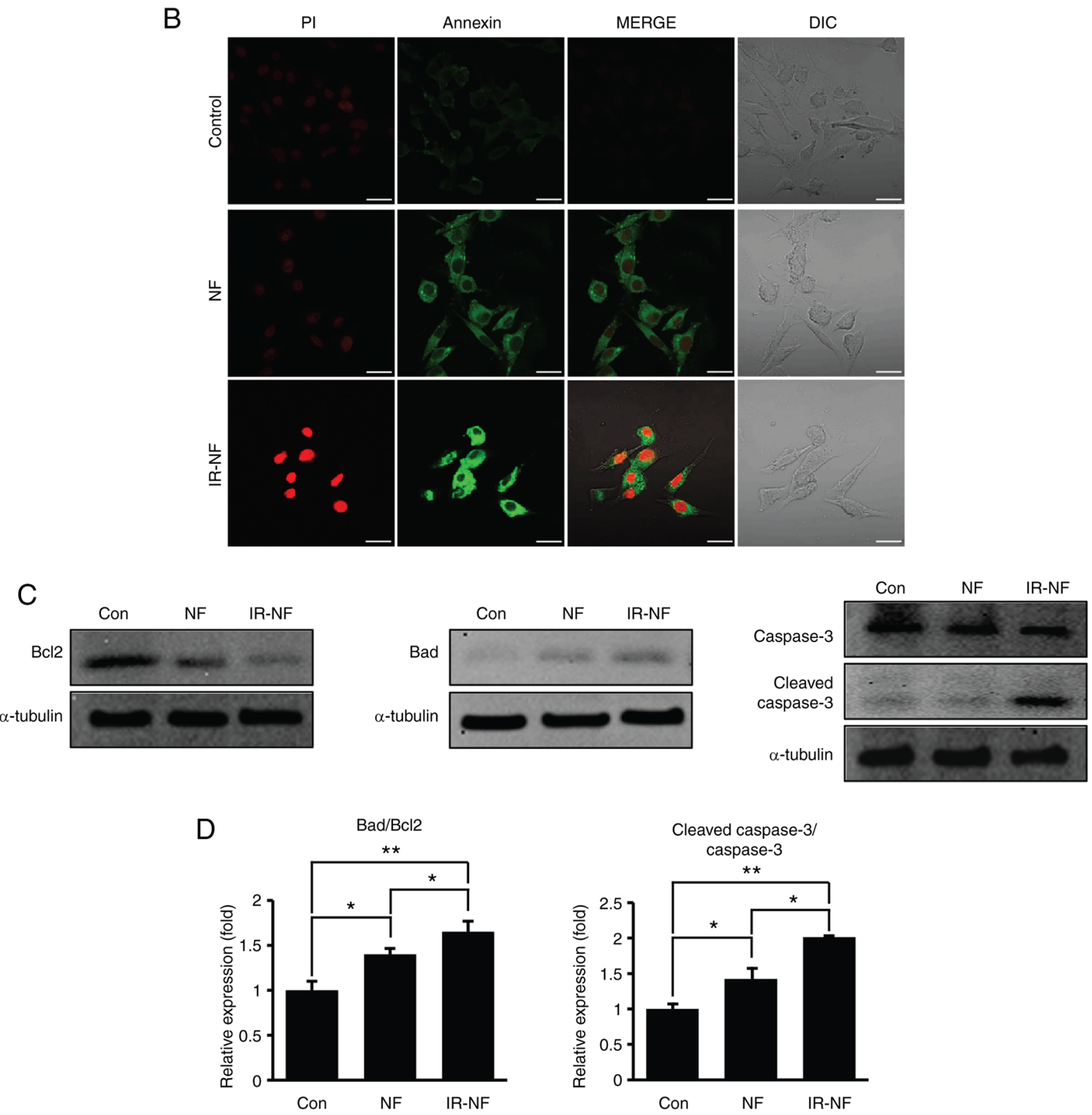

Figure 2. IR-NF induces apoptosis in the MCF-7 cell line. MCF-7 cells were treated with the indicated doses of NF or IR-NF for 24 h. Cells were stained with Annexin V-fluorescein isothiocyanate and analyzed using (A) a cell sorting system with Muse and (B) confocal microscopy (magnification, $\mathrm{x} 40$ ). (C) Whole cell lysates prepared and immunoblotted with anti-anti-apoptotic B cell lymphoma 2, anti-BAD, anti-caspase 3 and anti-cleaved caspase 3 . Anti- $\alpha$-tubulin was used as a loading control. (D) The western blots were quantitatively analyzed. Band intensities were normalized to those of the normal form of each protein or $\alpha$-tubulin. Data are presented as the mean \pm standard deviation. ${ }^{*} \mathrm{P} \leq 0.05,{ }^{* *} \mathrm{P} \leq 0.01$ vs. NF. IR-NF, irradiated nomifensine; NF, nomifensine; Con, control; DIC, differential interference contrast.

as an anticancer agent (4). A translational approach to medical drug candidates with pharmacological effects is as valuable as discovering new drug candidates. However, even if the drug is used for other purposes, the concerns of previously reported reversible effects cannot be ignored. As there is controversy over the application of NF in humans, there might be an alternative strategy that reduces the dosage in humans by increasing the efficacy of the pharmacological effect of NF. 
A

Con

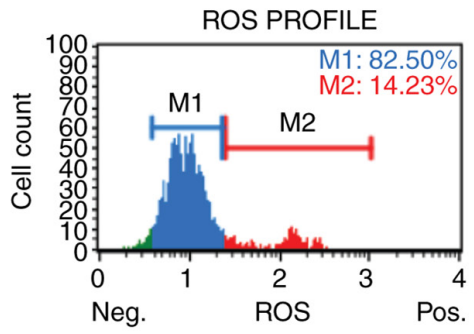

B

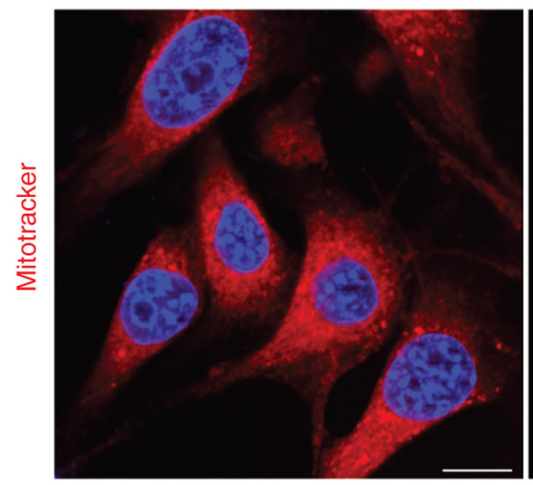

NF

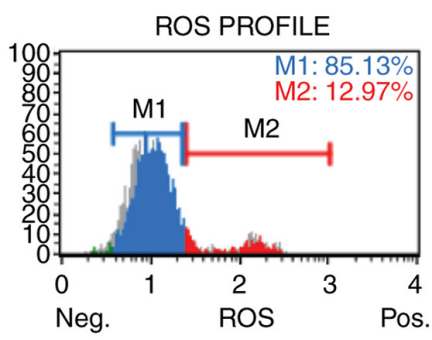

NF

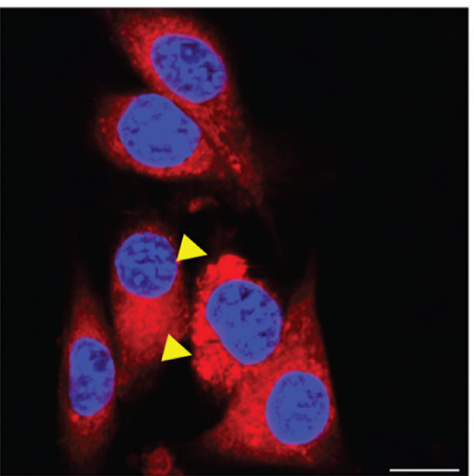

IR-NF

ROS PROFILE

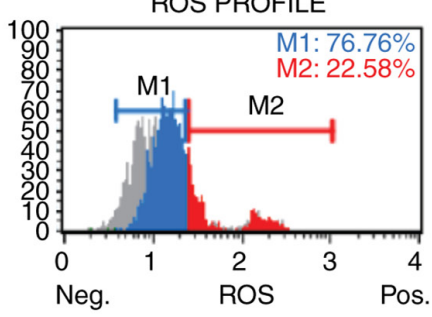

IR-NF

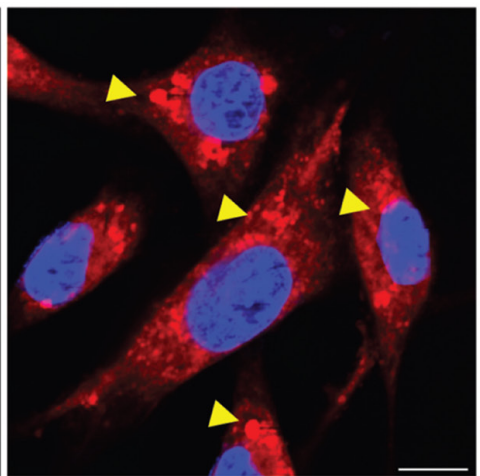

Figure 3. IR-NF induced ROS accumulation and morphological changes of the mitochondria. MCF-7 cells were treated with the indicated doses of NF or IR-NF for 6 h. (A) Intracellular ROS detection using the Muse Cell Analyzer. (B) Representative images of Mitotracker staining and MCF-7 cells were cultured on glass coverslips; the cells were fixed and the nuclei were counterstained with 4',6-diamidino-2-phenylindole. The yellow arrows indicate the swollen mitochondria. Scale bar $=10 \mu \mathrm{m}$. IR-NF, irradiated nomifensine; ROS, reactive oxygen species; NF, nomifensine; Con, control.
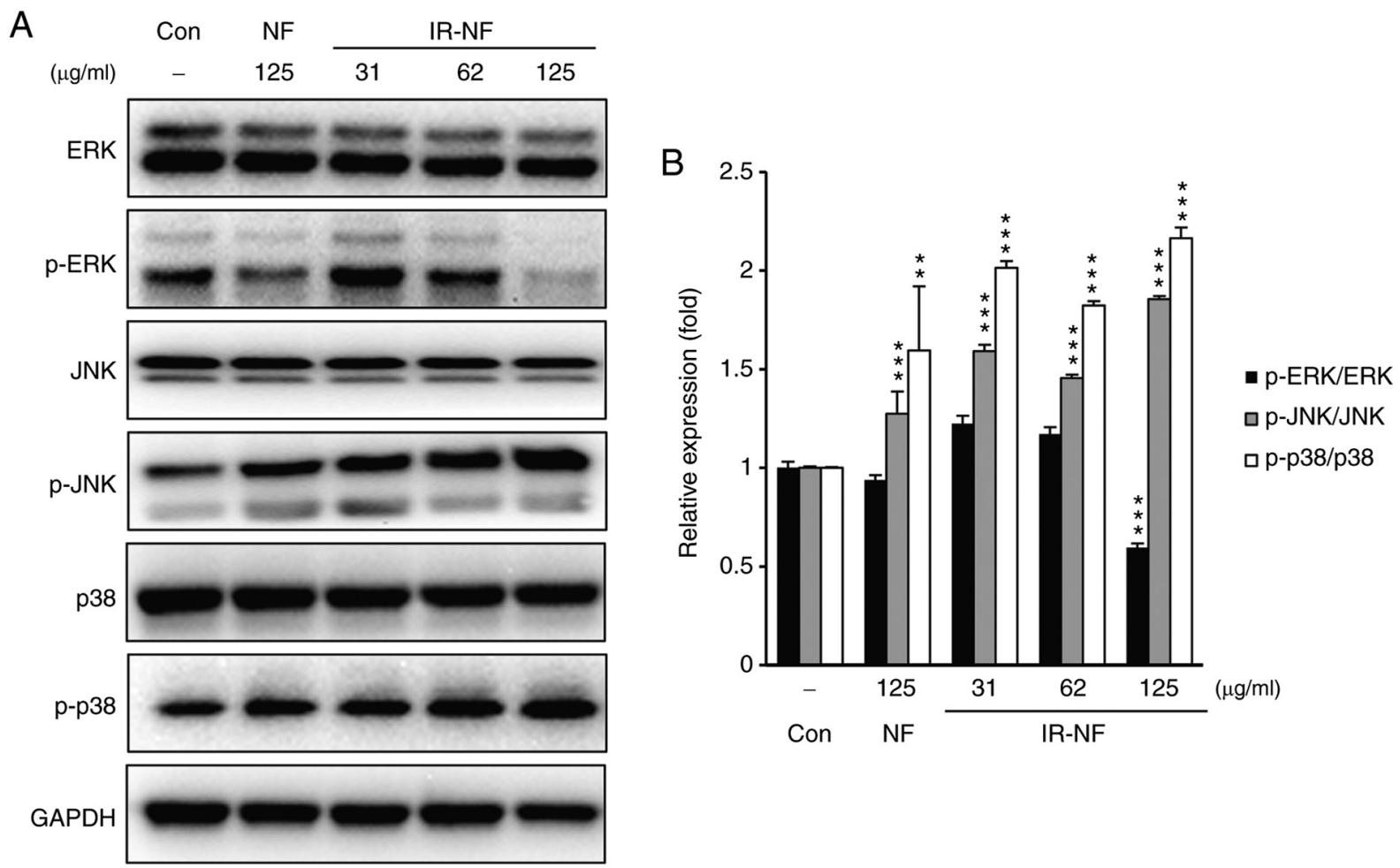

Figure 4. IR-NF stimulates the MAPK pathway in MCF-7 cells. (A) Representative images of western blot analysis for the protein expressions of JNK, p-JNK, p38, p-p38, ERK, p-ERK and glyceraldehyde-3-phosphate dehydrogenase antibodies. (B) Densitometry analysis. The data are presented as the mean \pm standard deviation. ${ }^{* *} \mathrm{P} \leq 0.01$ and ${ }^{* * *} \mathrm{P} \leq 0.001$ vs. control. IR-NF, irradiated nomifensine; MAPK, mitogen-activated protein kinase; JNK, c-Jun NH 2-terminal kinase; p-, phosphorylated; ERK, extracellular signal-regulated kinase; NF, nomifensine; Con, control.

The present study explored the anticancer mechanism of NF and found that IR increased its efficacy.
IR was used to change the chemical properties of NF. The main peak of NF decreased following IR, which occurred 


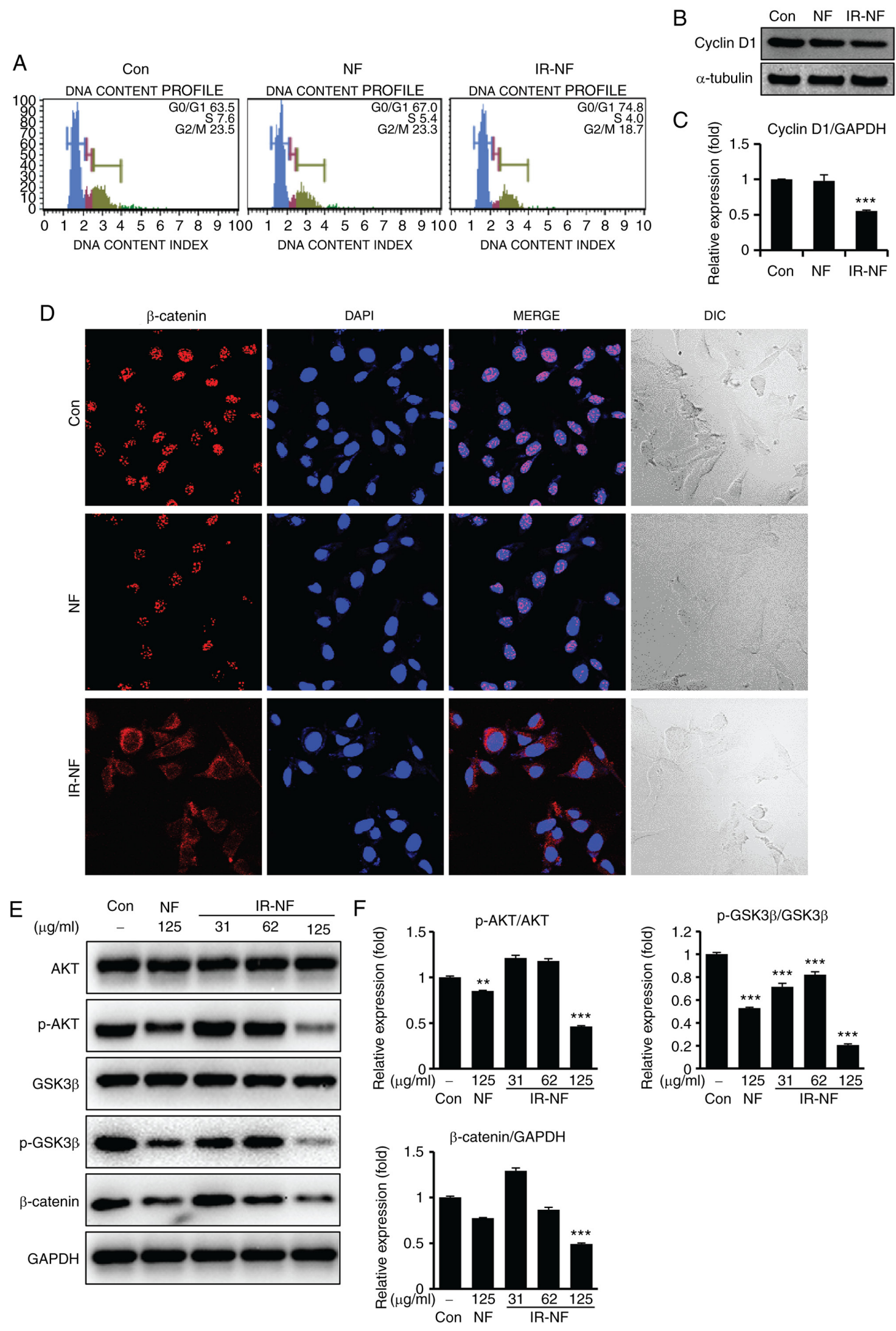

Figure 5. IR-NF induced cell cycle arrest and suppressed $\beta$-catenin translocation to the nucleus in MCF-7 cells. MCF-7 cells were treated with IR-NF $(125 \mu \mathrm{g} / \mathrm{ml})$ for $24 \mathrm{~h}$. (A) Cells were stained using the Cell Cycle Assay Kit and analyzed using the Muse cell sorting system. (B) Whole cell lysates prepared and immunoblotted with anti-cyclin B1. Anti- $\alpha$-tubulin was used as loading controls. (C) Densitometry analysis. Data are presented as the mean \pm standard deviation. ${ }^{* * *} \mathrm{P} \leq 0.001$ vs. control. (D) Cells were stained with anti- $\beta$-catenin antibody and observed using confocal microscopy. Scale bar $=25 \mu \mathrm{m}$. (E) Whole cell lysates were prepared and immunoblotted with anti-AKT, anti-p-AKT, anti-GSK3 $\beta$, anti-p-GSK3 $\beta$, anti- $\beta$-catenin and anti-GAPDH were used as loading controls. (F) The western blots were quantitatively analyzed. Band intensities were normalized to those of the normal form of each protein or GAPDH. Data are presented as the mean \pm standard deviation. ${ }^{* *} \mathrm{P} \leq 0.01,{ }^{* * * *} \mathrm{P} \leq 0.001$ vs. Con. IR-NF, irradiated nomifensine; IR-NF, irradiated

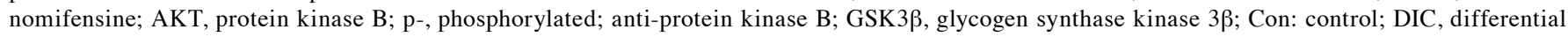
interference contrast. 
simultaneously with the detection of novel peaks. IR produced marked modifications in the chemical properties of NF. In addition, as a result of conducting a toxicity evaluation on several cancer cell lines (A549, SK-MEL-5, MCF-7) using NF, it was confirmed that the toxicity was observed only in the MCF-7 cell line. In addition to a previous report which stated that NF can inhibit cell growth in breast cancer cells, it was confirmed that the change in the characteristics of NF induced by irradiation enhanced its anti-proliferative efficacy (5).

The present study demonstrated that IR-NF effectively inhibited cell proliferation at a lower concentration compared with NF. IR-NF dysregulated mitochondrial formation, increased ROS accumulation, induced caspase 3 dependent apoptosis, stimulated MAPK (ERK, JNK, p38) pathways and suppressed the cell cycle.

Mitochondria serve an important role in triggering and regulating apoptosis (20). In particular, regulation of the cellular balance between pro- and anti-apoptotic mitochondrial proteins is pivotal for the determination of cell fate by either promoting survival or mitochondrial-mediated apoptosis (21). Under stressful conditions, such as intracellular ROS, physical/chemical stimuli, mitochondrial deoxyribonucleic acid damage and hypoxia, mitochondrial membrane permeability and release of pro-apoptotic proteins from the intermembrane space of the mitochondria into the cytosol increases $(22,23)$. In addition, stresses due to these various internal/external stimuli increase the cellular expression of pro-apoptotic proteins relative to anti-apoptotic proteins, inducing apoptosis (21). From this point of view, the data suggested that IR-NF treatment in MCF-7 cells induced apoptosis by inducing functional damage to the mitochondria.

On the other hand, the present study demonstrated that IF-NF upregulated the phosphorylation of JNK and p38 proteins and downregulated the phosphorylation of the ERK protein in MCF-7 cells. The MAPK pathway serves a crucial role in most cellular functions and can mediate different anti-proliferative events, such as apoptosis, autophagy and senescence, depending on the cell type and stimulus (24-27). In a number of previous studies, it has been suggested that MAPKs (ERK1/2, p38 and JNK) are involved in inhibiting the onset of breast tumors, inhibiting tumor metastasis and being an implicit parameter for sensitization to tumor suppression and apoptosis (28-30). Additionally, previous studies reported that activation of Wnt signaling increases the transcription of a number of genes, including cyclin D1, in breast cancer $(31,32)$. Overexpression of cyclin D1 occurs in $>50 \%$ of breast cancers (33). Taken together, the results showed trends similar to those of previous studies, suggesting that MAPK pathways and $\beta$-catenin signaling are involved in the apoptosis of MCF-7 cells caused by IR-NF treatment.

In the present study, IR was used because of its potential to introduce energy into a material to create new polarity or characteristics. In addition, materials irradiated with sufficiently high energy can decompose, creating highly reactive intermediate molecules or forming new molecules. However, the varying possibilities of material variations induced by IR can raise concerns about reproducibility. For irradiation technology to be used as a new platform for drug development, repetitive reproducibility and yield will be an important issue. In a pre-experiment of the present study, it was found that the reproducibility of radiolytic $\mathrm{NF}$ and its derivatives were represented when NF solutions with different concentrations and volumes were irradiated by IR at a total dose of $75 \mathrm{kGy}$. This indicated that gamma irradiation is an effective way to produce NF derivatives with improved efficacy and has provided new insights into the development of effective new drugs. The present study investigated the anticancer effects of NF derivatives on breast cancer cells and demonstrated that the potential effects of these derivatives serve an important role in the inhibition of MCF-7 proliferation. Although not all derivatives were isolated, it would be a valuable discovery if some derivatives showed improved cytotoxicity in one compound compared with the original material.

In conclusion, the present study provided the first evidence that IR-induced modification significantly contributed to improving the pharmacological properties and anticancer efficacy of NFs for the treatment of breast cancer. The results will provide an opportunity for re-evaluation of a number of drugs whose use has been restricted due to serious adverse effects or drug resistance. Although the present study reached meaningful conclusions from cellular-level studies, the diversity of environments in humans and animals is insufficient to make a strong claim that IR-NF is a valuable cancer-associated marker. The physiological effects of IR-NF on the human will be meaningful through animal and clinical studies and will be clarified through ongoing research. In addition, separating individual fractions to analyze key single compounds and confirm their effectiveness will have to be carried out in further studies.

\section{Acknowledgements}

Not applicable.

\section{Funding}

The present study was supported by Convergence Research Group project (grant no. CRC21021-300) of the National Research Council of Science and Technology, Republic of Korea.

\section{Availability of data and materials}

The datasets used and/or analyzed during the current study are available from the corresponding author on reasonable request.

\section{Authors' contributions}

SHK conceived the present study and wrote the original draft. DHB performed formal analysis and investigation. HWB and BYC performed the statistical analysis and revised the manuscript critically for important intellectual contents. SHK and DHB confirmed the authenticity of all the raw data. All authors read and approved the final manuscript.

\section{Ethics approval and consent to participate}

Not applicable. 


\section{Patient consent for publication}

Not applicable.

\section{Competing interests}

The authors declare that they have no competing interests.

\section{References}

1. Sledge GW, Mamounas EP, Hortobagyi GN, Burstein HJ, Goodwin PJ and Wolff AC: Past, present and future challenges in breast cancer treatment. J Clin Oncol 32: 1979-1986, 2014

2. Razak NA, Abu N, Ho WY, Zamberi NR, Tan SW, Alitheen NB Long $\mathrm{K}$ and Yeap SK: Cytotoxicity of eupatorin in MCF-7 and MDA-MB-231 human breast cancer cells via cell cycle arrest anti-angiogenesis and induction of apoptosis. Sci Rep 9: 1514, 2019.

3. Wang $\mathrm{X}$, Zhang $\mathrm{H}$ and Chen $\mathrm{X}$ : Drug resistance and combating drug resistance in cancer. Cancer Drug Resist 2: 141-160, 2019.

4. Tong XY, Quan Y and Zhang HY: NUDT5 as a novel drug target and prognostic biomarker for ER-positive breast cancer. Drug Discov Today 26: 620-625, 2021.

5. Tong XY, Liao X, Gao M, Lv BM, Chen XH, Chu XY, Zhang QY and Zhang HY: Identification of NUDT5 inhibitors from approved drugs. Front Mol Biosci 7: 44, 2020.

6. Kinney JL: Nomifensine maleate: A new second-generation antidepressant. Clin Pharm 4: 625-636, 1985.

7. Robinson S, Cheney D and Costa EJN-Ssaop: Effect of nomifensine and other antidepressant drugs on acetylcholine turnover in various regions of rat brain. Naunyn Schmiedebergs Arch Pharmacol 304: 263-269, 1978

8. Katz NS, Guiard BP, El Mansari M and Blier P: Effects of acute and sustained administration of the catecholamine reuptake inhibitor nomifensine on the firing activity of monoaminergic neurons. J Psychopharmacol 24: 1223-1235, 2010.

9. CSM Update: Withdrawal of nomifensine. Br Med J (Clin Res Ed) 293: 41, 1986

10. Böning J and Fuchs G: Nomifensine and psychological dependence-a case report. Pharmacopsychiatry 19: 386-388, 1986.

11. Badaboina S, Bai HW, Na YH, Park CH, Kim TH, Lee TH and Chung BY: Novel radiolytic rotenone derivative, rotenoisin B with potent anti-carcinogenic activity in hepatic cancer cells. Int J Mol Sci 16: 16806-16815, 2015.

12. Lee EH, Park CH, Choi HJ, Kawala RA, Bai HW and Chung BY: Dexamethasone modified by gamma-irradiation as a novel anticancer drug in human non-small cell lung cancer. PLoS One 13: e0194341, 2018.

13. Bak DH, Kang SH, Park CH, Chung BY and Bai HW: A nove radiolytic rotenone derivative, rotenoisin $\mathrm{A}$, displays potent anticarcinogenic activity in breast cancer cells. J Radiation Res 62 249-258, 2021

14. Kawala RA, Ramadhani FJ, Choi HJ, Lee EH, Park CH, Chung BY and Bai HW: Kenalog modified by ionizing radiation induces intrinsic apoptosis mediated by elevated levels of reactive oxygen species in melanoma cancer. Oncol Rep 41: $1837-1850,2019$.

15. Ježek J, Cooper KF and Strich R: Reactive oxygen species and mitochondrial dynamics: The Yin and Yang of mitochondrial dysfunction and cancer progression. Antioxidants (Basel) 7: 13 2018.
16. Derin D, Eralp Y, Ozluk Y, Yavuz E, Guney N, Saip P, Igci A, Ozmen V, Kücücük S, Aslay I, et al: Lower level of MAPK expression is associated with anthracycline resistance and decreased survival in patients with hormone receptor negative breast cancer. Cancer Invest 26: 671-679, 2008.

17. Zhang $\mathrm{Y}$ and Wang $\mathrm{X}$ : Targeting the $\mathrm{Wnt} / \beta$-catenin signaling pathway in cancer. J Hematol Oncol 13: 165, 2020.

18. Yu WK, Xu ZY, Yuan L, Mo S, Xu B, Cheng XD and Qin JJ: Targeting $\beta$-catenin signaling by natural products for cancer prevention and therapy. Front Pharmacol 11: 984, 2020.

19. Bugter JM, Fenderico N and Maurice MM: Mutations and mechanisms of WNT pathway tumour suppressors in cancer. Nat Rev Cancer 21: 5-21, 2021.

20. Tait SWG and Green DR: Mitochondrial regulation of cell death. Cold Spring Harb Perspect Biol 5: a008706, 2013.

21. Redza-Dutordoir M and Averill-Bates DA: Activation of apoptosis signalling pathways by reactive oxygen species. Biochim Biophys Acta 1863: 2977-2992, 2016.

22. Zorov DB, Juhaszova M and Sollott SJ: Mitochondrial reactive oxygen species (ROS) and ROS-induced ROS release. Physiol Rev 94: 909-950, 2014.

23. Webster KA: Mitochondrial membrane permeabilization and cell death during myocardial infarction: Roles of calcium and reactive oxygen species. Future Cardiol 8: 863-884, 2012.

24. Yue J and López JM: Understanding MAPK signaling pathways in apoptosis. Int J Mol Sci 21: 2346, 2020.

25. Chang $\mathrm{H}$ and Zou Z: Targeting autophagy to overcome drug resistance: Further developments. J Hematol Oncol 13: 159, 2020

26. Cargnello M and Roux PP: Activation and function of the MAPKs and their substrates, the MAPK-activated protein kinases. Microbiol Mol Biol Rev 75: 50-83, 2011.

27. Munshi A and Ramesh R: Mitogen-activated protein kinases and their role in radiation response. Genes Cancer 4: 401-408, 2013.

28. Davidson B, Konstantinovsky S, Kleinberg L, Nguyen MT, Bassarova A, Kvalheim G, Nesland JM and Reich R: The mitogen-activated protein kinases (MAPK) p38 and JNK are markers of tumor progression in breast carcinoma. Gynecol Oncol 102: 453-461, 2006.

29. Whyte J, Bergin O, Bianchi A, McNally S and Martin F: Key signalling nodes in mammary gland development and cancer. Mitogen-activated protein kinase signalling in experimental models of breast cancer progression and in mammary gland development. Breast Cancer Res 11: 209, 2009.

30. Kawiak A, Domachowska A, Krolicka A, Smolarska M and Lojkowska E: 3-chloroplumbagin induces cell death in breast cancer cells through MAPK-mediated Mcl-1 inhibition. Front Pharmacol 10: 784, 2019.

31. He Y, Liu Z, Qiao C, Xu M, Yu J and Li G: Expression and significance of Wnt signaling components and their target genes in breast carcinoma. Mol Med Rep 9: 137-143, 2014.

32. Guo L, Yilamu D, Sun L, Liu S and Ma F: Association among the expression of $\beta$-catenin, cyclin D1 and estrogen receptor- $\beta$ in human breast cancer. Exp Ther Med 10: 1423-1428, 2015.

33. Velasco-Velázquez MA, Li Z, Casimiro M, Loro E, Homsi N and Pestell RG: Examining the role of cyclin D1 in breast cancer. Future Oncol 7: 753-765, 2011.

This work is licensed under a Creative Commons Attribution-NonCommercial-NoDerivatives 4.0 International (CC BY-NC-ND 4.0) License. 